\title{
Dominant negative effects of SCN5A missense
}

\section{variants}

4 Matthew J. O’Neill ${ }^{1}$, Ayesha Muhammad ${ }^{1}$, Bian Li², Yuko Wada², Lynn Hall², Joseph F. Solus ${ }^{2}$,

5 Laura Short², Dan M. Roden ${ }^{3}$, Andrew M. Glazer²*

\section{Affiliations}

1. Vanderbilt University School of Medicine, Nashville, TN

2. Vanderbilt Center for Arrhythmia Research and Therapeutics (VanCART), Division of Clinical Pharmacology, Department of Medicine, Vanderbilt University Medical Center, Nashville, TN

3. Vanderbilt Center for Arrhythmia Research and Therapeutics (VanCART), Departments of Medicine, Pharmacology, and Biomedical Informatics, Vanderbilt University Medical

15

16 Correspondence should be addressed to Andrew Glazer, PhD, Vanderbilt University Medical

17 Center, 2215B Garland Ave, 1235J Medical Research Building IV, Nashville, TN 37232. Email: 


\section{Abstract}

26 Introduction: Up to $30 \%$ of patients with Brugada Syndrome (BrS) carry loss-of-function (LoF)

27 variants in the cardiac sodium channel gene SCN5A. Recent studies have suggested that the

28 SCN5A protein product Nav1.5 can form dimers and exert dominant negative effects.

29 Methods: We identified 35 LoF variants (<10\% peak current compared to wild type (WT)) and 15

30 partial LoF variants (10-50\% peak current compared to WT) that we assessed for dominant

31 negative behavior. SCN5A variants were studied in HEK293T cells alone or in heterozygous co-

32 expression with WT SCN5A using automated patch clamp. To assess clinical risk, we compared

33 the prevalence of dominant negative vs. putative haploinsufficient (frameshift/splice site) variants

34 in a BrS case consortium and the gnomAD population database.

35 Results: In heterozygous expression with WT, 32/35 LoF variants and 6/15 partial LoF showed

36 reduction to $<75 \%$ of $\mathrm{WT}$-alone peak $I_{\mathrm{Na}}$, demonstrating a dominant negative effect. Carriers of

37 dominant negative LoF missense variants had an enriched disease burden compared to putative

38 haploinsufficient variant carriers (2.7-fold enrichment in BrS cases, $p=0.019)$.

39 Conclusions: Most SCN5A missense LoF variants exert a dominant negative effect. Cohort 40 analyses reveal that this class of variant confers an especially high burden of BrS. 


\section{Introduction}

Brugada Syndrome $(\mathrm{BrS})$ is a clinical arrhythmia syndrome with characteristic EKG

44 changes in the absence of underlying structural heart abnormalities (1). While often asymptomatic

45 or clinically unrecognized, sudden cardiac death (SCD) due to ventricular tachyarrhythmia can be

46 the sentinel manifestation. Up to $30 \%$ of BrS patients have heterozygous loss-of-function (LoF)

47 variants in the cardiac sodium channel gene SCN5A, which encodes the channel protein Nav1.5

48 (2). A recent evaluation by ClinGen asserted that SCN5A was the only gene with strong evidence

49 for Mendelian associations with BrS (3). LoF SCN5A variants are also associated with other

50 arrhythmias including sick sinus syndrome (4) and progressive cardiac conduction disease (5).

Over 100 LoF variants within SCN5A have been reported across multiple variant classes

52 including missense, nonsense, splice-altering, and frameshift/premature truncation (2, 6). SCN5A

53 encodes a channel with 4 transmembrane domains, each consisting of 6 transmembrane

54 segments (7). Nav1.5 has traditionally been thought to function as a monomer; however, a recent

55 study indicated that Nav1.5 can form dimers with coupled intracellular trafficking and/or gating at

56 the plasma membrane (8). Similar to variants in established multimeric proteins that can generate

57 dominant negative effects, several missense SCN5A variants with dominant negative effects on

58 trafficking or coupled gating at the cell surface have been reported in vitro and in vivo (9-11).

59 However, the dominant negative behavior of most of the approximately 40 known LoF missense

60 variants in SCN5A has not been tested. Moreover, the degree of dominant negative effects among

61 partial LoF missense variants has not been evaluated.

62 Variable penetrance is a hallmark of pathogenic BrS variants, and the extent to which

63 distinct pathogenic mechanisms (e.g., dominant negative vs haploinsufficiency) contribute to this

64 effect is unknown. Large cohort studies and variant curation efforts provide datasets of SCN5A

65 variants associated with $\operatorname{BrS}$ cases $(2,6,12)$. In addition, large population cohorts such as

66 gnomAD provide sets of individuals more likely representing putative controls (13). Together,

67 these datasets enable the comparison of BrS disease risk among different variant classes. 
bioRxiv preprint doi: https://doi.org/10.1101/2021.09.22.461398; this version posted September 24, 2021. The copyright holder for this preprint (which was not certified by peer review) is the author/funder, who has granted bioRxiv a license to display the preprint in perpetuity. It is made available under aCC-BY-ND 4.0 International license.

69 partial LoF missense variants. We use case and control cohorts to test the relative BrS disease

70 risk of dominant negative missense variants compared with other variant classes.

71 


\section{Methods}

73 Selection of Variants. Variants for this study were selected from previously published functionally

74 characterized variants $(6,14)$. Variants with peak currents $<10 \%$ compared to WT were

75 considered LoF, and variants with peak currents between 10-50\% compared to WT were

76 considered partial LoF. A full list of variants in this study is presented in Supplementary Table 1.

78 SCN5A Mutagenesis. The SCN5A variant plasmids were mutagenized using a previously 79 described "zone" system (14). Briefly, SCN5A individual zones on small plasmids were 80 mutagenized using the QuikChange Lightning Multi kit (Agilent) with primers designed using the

81 online QuikChange Primer Design tool. Primers used in this study are listed in Supplementary

82 Table 2. The variant-containing zone was then subcloned by restriction digestion into a plasmid 83 containing an AttB:SCN5A:IRES:mCherry-blasticidinR plasmid (14-16). The entire sequence of

84 the zone containing the variant was confirmed by Sanger sequencing. In a previous study of 82

85 variants generated by this approach, 0/82 plasmids had any additional SCN5A mutations outside

86 the target zone (14). All analyses used the most common SCN5A transcript in the adult heart,

87 including the adult isoform of exon 6 and a deletion of the alternatively spliced GIn1077 residue

88 (ENST00000443581). As per convention, all variants are named in accordance with the full 2,016

89 amino acid form (ENST00000333535).

91 Description of Cell Lines: All experiments used Human Embryonic Kidney HEK293T "negative

92 selection" landing pad (LP) cells as previously described (gift of Kenneth Matreyek) (14-16). The

93 AttB/AttP LP allows a single integration event per cell and a consistent level of target gene

94 expression (Figure 1). Homozygous experiments were carried out in LP cells (Figure 1A).

95 Plasmids carrying SCN5A variants were transfected along with transposase and integrated into

96 the LP site to allow stable expression. We termed these lines LP-SCN5A. 
97 For heterozygous expression, we first generated LP cells stably expressing WT SCN5A in a non-

98 LP site using the Sleeping Beauty (SB) transposon system and identified a clone of this cell line 99 with peak sodium current ( $\left.\mathrm{I}_{\mathrm{Na}}\right)$ equivalent to that observed with WT SCN5A in the LP site (Figure 100 1B). We then generated cell lines with SCN5A variants transfected into the LP site, thereby 101 allowing us to express the two SCN5A alleles at equivalent levels and assess dominant negative 102 effects. These lines are referred to as LP-SB-SCN5A.

104 Generation of Cell Lines: Cells were cultured at $37^{\circ} \mathrm{C}$ in humidified $95 \%$ air $/ 5 \% \mathrm{CO}_{2}$ incubator in 105 “HEK media”: Dulbecco's Eagle's medium supplemented with $10 \%$ fetal bovine serum, $1 \%$ non106 essential amino acids, and 1\% penicillin/streptomycin. Stable integration of a WT SCN5A into LP107 cells was achieved using an optimized SB transposon system (17) using the pSBbi-GN plasmid 108 (a gift from Eric Kowarz, Addgene \#60517), which contains SB transposon sequences for genomic integration flanking a promoter upstream of GFP and a second promoter upstream of a multiple cloning site (MCS) for expression of a gene of interest. A Notl restriction site was first 111 cloned into the multiple cloning site using Gibson assembly (New England Biolabs). Then, WT 112 SCN5A was cloned into the MCS by Notl digestion (New England Biolabs). Next, 1 ug of 113 pSBbiGN-SCN5A and $100 \mathrm{ng}$ of pCMV(CAT)T7-SB100, a plasmid expressing SB transposase (a 114 gift from Zsuzsanna Izsvak, Addgene \#34879), were cotransfected into the cells (18), using 115 FuGENE 6 (Promega) following manufacturer's instructions. At day 7 post-transfection, GFP+ 116 cells were sorted by fluorescence-activated cell sorting (FACS), and individual colonies were 117 picked and re-analyzed by analytical flow cytometry to identify clones expressing varying levels 118 of GFP (and thus varying levels of $\mathrm{Nav}_{1}$.5). Clones were then tested by SyncroPatch automated 119 patch clamping (see below) to identify a clone expressing an equal peak sodium current as results 120 from typical integration of a single copy of wild-type Nav1.5 into the AttB/AttP landing pad.

121 For homozygous patch clamp experiments, LP cells were transfected with an AttB-SCN5A 122 variant:IRES:mCherry-BlasticidinR plasmid and studied as previously described (14). For 
123 heterozygous patch clamp experiments, LP-SB-SCN5A cells were transfected using similar

124 methods. For all cell lines, cells were transfected with FuGENE 6 or Lipofectamine 2000 following

125 manufacturer's suggested protocols using an AttB-containing SCN5A:IRES:mCherry:blasiticidinR

126 plasmid and a plasmid bearing Bxb1 recombinase; cells underwent negative selection for 6 days

127 with $1 \mathrm{ug} / \mathrm{mL}$ doxycycline (to induce promoter expression; Sigma), $100 \mathrm{ug} / \mathrm{mL}$ blasticidin $\mathrm{S}$ (to kill

128 cells not expressing the blasticidin-resistant plasmid; Sigma), and 10 nM AP1903 (to kill un-

129 integrated cells expressing the AP1903-sensitive caspase gene; MedChemExpress) in HEK

130 media (15). At the end of selection, cells were assessed by analytical flow cytometry to assess

131 percentage of mCherry-positive, BFP-negative cells (LP integration of SCN5A variant) and GFP-

132 positive cells (SB integration of SCN5A).

134 Automated Patch Clamping. Electrophysiology data were collected with the SyncroPatch 384PE 135 automated patch clamping device (Nanion) using the same cell preparation and solutions as 136 previously reported (14). Peak currents are reported at -20 mV after a $200 \mathrm{msec}$ pulse from a 137 resting potential of $-120 \mathrm{mV}$; peak sodium current is presented as the mean of data obtained in $138 \geq 8$ cells/variant (homozygous experiments) or $\geq 27$ cells/variant (heterozygous experiments). 139 Voltage of half activation, voltage of half inactivation, time of $50 \%$ recovery from inactivation, and 140 late current at $200 \mathrm{~ms}$ were obtained using previously published protocols (14). As previously 141 described, cells with values greater than 2.5 standard deviations from the mean were removed in 142 an automated process (14). For these additional parameters, only variants with data collected 143 from $>10$ cells were included.

145 Case-control analysis. We performed case-control analyses to test the penetrance of different 146 classes of variants. We used BrS case counts from a recent International BrS Genetics 147 Consortium and putative controls from gnomAD; the frequency of these variants is presented in 148 Supplementary Table $1(12,13)$. We use gnomAD as putative controls; although phenotypes are 
not available for gnomAD participants, the vast majority of these individuals should not have Brugada Syndrome. All gnomAD counts were taken from gnomAD v2.1.1 transcript ENST00000333535.4. A cut-off minor allele frequency of $2.5 \mathrm{e}-5$ was used to designate ultra-rare variants, as previously suggested (19). To test the severity of each disease class (i.e., missense vs. indel vs. splice/frameshift/nonsense), we compared the relative number of cases versus

controls by variant, drawing from the BrS consortium and gnomAD. Frameshift, splice, and nonsense variants at amino acid position > 1800 (post-transmembrane domain IV) were excluded due to the possibility that these variants may not be full LoF. We calculated the odds ratio

157 associated with each variant class according to the formula $(a / b) /(c / d)$, where $a=B r S$ cases with

158 variant, $b=$ BrS cases without variant, $c=$ gnomAD controls with variant, and $d=$ gnomAD controls

159 without variant. Since the allele number varied for different variants in gnomAD, the average allele

160 number was calculated over all relevant mutation types (missense, frameshift, nonsense, and

161 splice site) and divided by 2 to obtain a count of sequenced gnomAD participants to use in odds

162 ratio calculations, following a previously published approach (12).

Data Analysis. SyncroPatch 384PE data were analyzed as previously reported (14). Peak current densities were calculated by dividing peak current at $-20 \mathrm{mV}$ by cell capacitance. For homozygous experiments, peak current densities were normalized to peak current densities observed in cells expressing WT plasmid. For heterozygous experiments, peak current densities were normalized

168 to that observed in LP-SB-SCN5A cells, i.e., those expressing a single WT allele. As described 169 below, WT+WT cells displayed $\sim 200 \%$ peak $I_{\mathrm{Na}}$ compared to LP-SB-SCN5A cells. Heterozygote 170 (WT+variant) cells displaying $<75 \%$ of peak $I_{\mathrm{Na}}$ compared to LP-SB-SCN5A cells were designated 171 as exerting a dominant negative effect. Statistical comparisons were made using two-tailed 172 Fisher's exact tests, implemented in R Studio (version 1.3.1093). 
174 Structural Analysis: Nav1.5 variant locations were determined from UniProt (20). The structural

175 model of human SCN5A (UniProtKB: Q14524-1, modeled residues: 30-440, 685-957, 1174-

176 1887) was generated by homology modeling using the protein structure prediction software

177 Rosetta (v.3.10) (21). The cryo-EM structure of human SCN9A bound with SCN1B and the Ig

178 domain of SCN2B resolved to $3.2 \AA$ (PDB: $6 \mathrm{~J} 8 \mathrm{H})(22)$ were used as the primary templates while

179 the cryo-EM structure of NavPaS from American Cockroach resolved to $2.6 \AA$ (PDB: 6A95) (23)

180 was used as a secondary template. The percent identity between the aligned positions of SCN9A

181 and SCN5A sequences is $76.7 \%$. While the percent identity between NavPaS and SCN5A was

182 only moderate (45.6\%), the $\mathrm{N}$-terminal and C-terminal domains in the NavPaS structure were

183 partially resolved, providing coordinates for modeling the corresponding domains of SCN5A. For

184 further details, see our previous report (14). Recently, an experimental structure of SCN5A was

185 determined using cryo-EM technique at a resolution of $3.3 \AA$ (24). We note that the root-mean-

186 square distance between our model and the experimental structure over all backbone atoms is

$1872.3 \AA$ (Supplementary Figure 1), suggesting that our model is accurate while covering more

188 residues than the experimental structure. 


\section{Results}

\section{1}

192 effect (Table S1).

\section{Homozygous and Heterozygous Measurements of LoF Variants}

We generated 37 LP-SCN5A stable lines (1 SCN5A allele expressed/line), each expressing LoF variants or the nonsense variant W822X (Figure 1, 2A and Table S1) $(15,16)$. Representative traces for WT and A735E are shown in Figure 2B. We recorded peak $I_{\mathrm{Na}}$ at -20 mV: $35 / 37$ missense variants exhibited a peak current density $<10 \%$ compared to WT (Figure $2 \mathrm{C}$ - only LoF shown; Supplementary Table 1). The remaining 2 variants (previously reported to be LoF) showed $>10 \%$ peak current when compared to WT and were studied separately with other partial LoF variants. One LoF variant, R893C, was previously detected in patients with BrS but has not been previously assessed by patch clamping (2).

We then tested each LoF variant in heterozygous expression (WT+variant) (Figure 1B and 2D). Figure $2 E$ shows representative traces of cells expressing $W T, W T+W T$, and $W T+A 735 E$ (an example dominant negative variant). Figure $2 \mathrm{~F}$ presents peak $\mathrm{I}_{\mathrm{Na}}$ for the same $35 \mathrm{LoF}$ variants presented in Figure 2C. WT+WT cells expressed peak $I_{\mathrm{Na}}$ of $218.4 \pm 7.7 \%$ relative to $\mathrm{WT}$ alone in LP-SB-SCN5A cells, i.e., those expressing a single WT allele. By contrast, 32/35 of the WT+variant cell lines showed $<75 \%$ peak $I_{\mathrm{Na}}$ compared to LP-SB-SCN5A cells, indicating a dominant negative effect. The heterozygous dominant negative variants displayed a gradient of effect, from $13.9 \pm 3.3 \%$ to $74.4 \pm 5.4 \%$ of WT alone. Two previously studied dominant negative variants, R104W and R121W (25), both also exhibited dominant negative effects in this study (69.6 $\pm 7.3 \%$ and $52.7 \pm 8.4 \%$ of WT, respectively). While W822X, G1661R, S1672Y, and R893C had LoF peak currents in homozygous experiments, they did not exhibit a dominant negative

\section{Homozygous and Heterozygous Measurements of Partial LoF Variants}


We also studied the prevalence of dominant negative effects in 15 partial LoF variants using LP-SB-SCN5A lines. We first confirmed that variant peak currents were 10\%-50\% compared to WT in homozygous expression with LP-SCN5A cells. (Figure 3A). The set of 15

217 variants included two variants (R282H and G1740R) previously reported to be LoF but measured 218 as $>10 \%$ peak $I_{\mathrm{Na}}$ in our system $(26,27)$. Figure $3 \mathrm{~B}$ shows a gradient of $I_{\mathrm{Na}}$, with partial LoF 219 variants showing a greater range of effect in heterozygous expression than those of LoF variants $220(24.7 \pm 5.6 \%$ to $231.6 \pm 10.8 \%$ ). 6/15 partial LoF variants had a dominant negative effect whereas

221 the remaining 9 variants all exceeded normalized WT peak current.

\section{Coupled gating in heterozygous expression}

In addition to assessing peak sodium current, we also examined other parameters of channel function to measure the extent of coupled gating, a phenomenon where the LoF allele alters the gating properties of the WT allele. These parameters required additional experimental

227 protocols and quality control filters, so these parameters were not comprehensively obtained in 228 all variants studied; only variants with data from $>10$ qualifying cells are presented. We examined 229 voltage of half activation among the missense variants investigated above (representative raw 230 data shown in Figure 4A and 4B). 16/50 variants (14 LoF and 2 partial LoF) showed a $>10 \mathrm{mV}$ 231 shift in the voltage of half activation, suggesting widespread coupled gating affecting this 232 parameter (Figure 4C). We did not observe widespread changes for other parameters beyond 233 voltage of half activation. No variants were shown to induce a shift in voltage of half inactivation $234>10 \mathrm{mV}$ (Figure 4D). One variant (G1406R) had a 1.71-fold change in recovery from inactivation 235 when compared to WT; the other 34 qualifying variants had $<50 \%$ shifts in RFI (Figure $4 \mathrm{E}$ ). No 236 variants induced late current $>1 \%$ when co-expressed with WT (Figure 4F). Due to the very low 237 or absent peak currents in homozygous LoF variants, it was not feasible to assess parameters 238 other than peak current in homozygous expression. 
Case and control counts of carriers of the dominant negative variants described above

242 were interrogated using a published consortia of BrS cases (12) and gnomAD, a database of

243 population variation that we considered to contain putative controls (13) (Figure 5A, Table S4). In

244 Figure 5B we present the odds ratios (ratio of odds in BrS cohort:gnomAD). The LoF missense

245 dominant negative variants had an odds ratio of 323 compared to 11.0 for missense, 24.2 for

246 indel, and 118 for putative haploinsufficient variants (nonsense, splice, frameshift). Thus, the

247 relative risk of dominant negative missense variants compared to haploinsufficient variants is 2.7

248 (Fisher's exact test, $p=0.019$ ). All categories were significantly enriched compared to all

249 missense variants (Fisher's exact test, $p<0.05)$.

250

\section{Structural Distribution of Dominant Negative Variants}

252 Dominant negative variants were present throughout the structured transmembrane 253 regions of Nav1.5 and did not predominate in any single hotspot region (Figure 6A). Structural 254 modeling further showed that dominant negative variants were distributed throughout the three255 dimensional structure of $\mathrm{Nav} 1.5$, with apparent enrichment in the S5-S6 linker domains (Figure 256 6B and 6C). 


\section{DISCUSSION}

\section{Dominant Negative Effect Among Most Missense LoF SCN5A Variants}

This study assessed the dominant negative properties of 50 LoF and partial LoF variants. dominant negative behavior. Dominant negative effects are pervasive throughout biology, especially for multimeric proteins, and involve several distinct mechanisms to compromise WT

264 function (28). In the case of $S C N 5 A$, the dominant negative effect has been posited to arise by

265 both deficient trafficking to the membrane as well as coupled gating at the cell surface. One study

266 showed that the variants $\mathrm{R} 104 \mathrm{~W}$ and $\mathrm{R} 121 \mathrm{~W}$ induced a dominant negative effect primarily

267 through endoplasmic reticulum retention of WT protein due to interactions among the channel

268 alpha-subunits (25). Follow up studies with extensive biochemical analyses showed that the dominant negative variant L325R acted through coupled gating at the cell surface (9).

Previous research suggested that the residues between 493 and 517 are critical for the

271 dimerization and coupled gating of Nav1.5 at the cell surface, and another study found an

272 enrichment of dominant negative variants at the $\mathrm{N}$-terminus of the protein $(8,29)$. We did not

273 observe an enrichment of dominant negative variants among these previously described residues,

274 but rather a broader distribution of variants spanning the four transmembrane domains of the

275 protein (Figure 6A-6C). Thus, dominant negative effects appear to be a general property of most

276 LoF missense variants in SCN5A, independent of location within the protein. Particularly

277 interesting are examples of disparate effects within close physical proximity, such as the partial

278 LoF variants V1405M (35.7\% peak current in heterozygous expression), V1405L (121\% peak 279 current), and G1406R (146\% peak current).

In addition to decreased peak current, we observed that $16 / 50$ variants also influenced

281 voltage of half activation when measured in heterozygous expression with WT. This finding is

282 consistent with the concept of coupled gating at the cell surface, and reflects the influence of the

283 loss of function allele on properties of the WT allele of the protein, possibly through a multi-channel 
complex (9). These shifts in V1/2 activation in a loss of function direction combine with reduced peak currents to result in additional reduction of channel function in heterozygous expression. $V_{1 / 2}$ activation was the only additional property that varied substantially from WT Nav1.5 activity, as we did not observe large differences in voltage of half inactivation, recovery from inactivation, or

288 late current.

\section{Increased BrS Risk of Dominant Negative Variants}

Previous work has established that homozygous peak current of SCN5A variants is the

292 strongest in vitro electrophysiological predictor of each variant's BrS risk (6, 30). Since dominant negative missense variants cause an especially low cellular peak current, we hypothesized that

294 dominant negative variants would confer an especially high risk for BrS. Importantly, our expanded catalog of 38 dominant negative SCN5A variants enabled us for the first time to calculate cohort-based estimates of disease risk of this class of variants. Using gnomAD and a recently published cohort of BrS cases $(12,13)$, we demonstrated that dominant negative variants are highly overrepresented in cases vs controls when compared to other variant classes, with a striking odds ratio of 323 for dominant negative LoF missense variants. In contrast, other variant classes have lower odds ratios of 11 (all missense variants) or 118 (putative haploinsufficient frameshift/nonsense/splice site variants). Thus, the relative risk of BrS among dominant negative LoF missense variants compared to putative haploinsufficient variants is 2.7. Previous studies have shown that truncating and functionally inactive missense variants cause a more severe 304 phenotype than partially active missense variants, but the penetrance of dominant negative 305 variants had not yet been extensively studied (31). Our results indicate that the penetrance of 306 dominant negative missense variants is higher than penetrance of other variant classes. One 307 potential explanation for the different disease penetrance among variant classes is that nonsense 308 mediated decay (NMD) removes aberrant transcripts for splice-altering and nonsense variants, 
preventing their interaction with WT Nav1.5. Given the data presented here, dominant negative missense variants should arouse high clinical suspicion for BrS risk when detected in patients.

\section{High-throughput Electrophysiological Assays to Study Dominant Negative Effects} functional consequences of ion channel genetic variation (32). This technique has been used to assess pathogenicity of variants in $\operatorname{KCNQ1}(33,34), \operatorname{SCN} 5 A(14)$, and $K C N H 2(35,36)$. Here, we present the most extensive evaluation of heterozygous Nav1.5 expression to date using this

317 platform, studying 51 variants with 27-164 cells per heterozygous measurement. Heterozygous

318 measurements are already common for the cardiac potassium channels $K C N Q 1$ and $K C N H 2$; this

319 study suggests that heterozygous studies may also be necessary for LoF SCN5A variants in future studies. This work shows that high-throughput automated patch-clamp can help establish

321 molecular mechanisms of disease.

Mechanistic and Therapeutic Implications

The prevalence of widespread dominant negative effects among SCN5A variants not only gives insight into action potential pathophysiology, but also provides a lead for therapeutic development. The multifunctional regulatory protein 14-3-3 has been reported to be critical for

327 mediating Nav1.5 dimerization, and an operative mechanism in select cases of the dominant negative effect (8). Indeed, difopein, an inhibitor of 14-3-3, (37) was shown to restore WT activity when co-expressed with dominant negative variants $(8,38)$. While targeting 14-3-3 may not be an appropriate therapeutic strategy given its role in myriad cellular processes, alternative

331 mechanisms to promote selective degradation of aberrant channels and preserve WT function

332 remain highly desirable. Emerging allele-specific siRNA or XNAzymes (39) strategies could ablate

333 the dominant negative effect prior to the translation event. A gene therapy approach has recently

334 been demonstrated for KCNQ1, and could be applied against dominant negative variants of BrS 
described here (40). Given the prevalence of the dominant negative phenomenon, and the high risk for BrS among carriers of these variants, there is a need for the development of novel

337 therapeutic strategies by leveraging basic biological insights.

\section{Limitations}

Results from heterologous expression in HEK293T cells may not fully recapitulate

341 behavior in native cardiomyocytes in human hearts. In particular, contributions such as polygenic

342 modifiers, as has been previously observed in BrS (41), may not be fully captured by this non-

343 native system. Two common alternative splicing events impact SCN5A splicing (Q1077

344 deletion/insertion and fetal/adult exon 6); only the most common splice isoform in the adult heart

345 was examined in this study. The gnomAD population database does not have available 346 phenotypic information, so a small fraction of individuals included in gnomAD may in fact have 347 BrS.

\section{Conclusions}

Most LoF missense variants in SCN5A have a dominant negative effect. These missense

351 dominant negative variants have a 2.7-fold increased risk of BrS when compared to putative 352 haploinsufficient variants. These results may help refine prediction of BrS risk in dominant 353 negative variant carriers.

\section{Acknowledgements}

356 We thank Victoria Parikh for helpful discussions, Kenneth Matreyek for suppling the LP-negative 357 HEK293 cell line, Eric Kowarz for supplying the pSBbi-GN plasmid, and Zsuzsanna Izsvak for 358 supplying the $\mathrm{pCMV}(\mathrm{CAT}) \mathrm{T7}$-SB100 plasmid. Flow Cytometry experiments were performed in the 359 VMC Flow Cytometry Shared Resource. The VMC Flow Cytometry Shared Resource is supported 
360 by the Vanderbilt Ingram Cancer Center (P30 CA68485) and the Vanderbilt Digestive Disease

361 Research Center(DK058404). SyncroPatch 384PE experiments were performed in the Vanderbilt

362 High-Throughput Screening (HTS) Core Facility. The HTS Core receives support from the

363 Vanderbilt Institute of Chemical Biology and the Vanderbilt Ingram Cancer Center (P30

364 CA68485).

365

366

367

368 Funding

369 This research was funded by NIH grants K99 HG010904 (AMG), R01 HL149826 (DMR),

370 T32GM007347 (MJO and AM), AHA grants AHA 20PRE35180088 (AM) and 20POST35220002

371 (BL), and a Heart Rhythm Society Clinical Research Award in Honor of Mark Josephson and Hein

372 Wellens (YW).

373

374

375 Disclosures

376 The authors report no conflicts and have no relevant disclosures. 


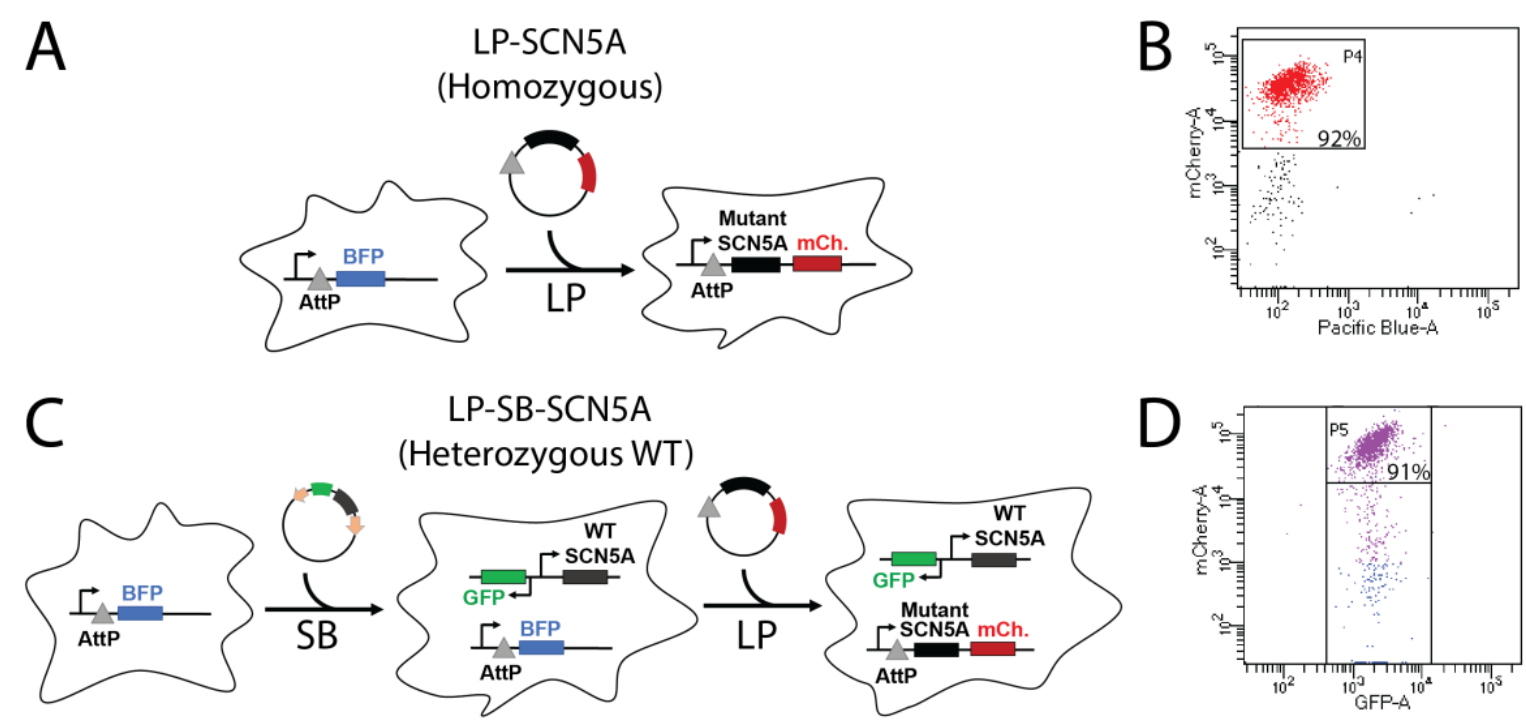

379

Figure 1. Stable cell lines used in this study. 1 or 2 copies of SCN5A were inserted into engineered HEK293 LP cells. The Landing Pad (LP) comprises an AttP and BFP locus, and allows insertion of a single insert per cell. A second Sleeping Beauty (SB) transposon system was used to introduce a second copy of the gene for heterozygous experiments.

A. Design of homozygous LP-SCN5A cell line with LP integration.

B. Analytical flow cytometry after incorporation of plasmid into the LP. Cells that do not have BFP expression and highly express mCherry (P4 gate) have a successful integration.

C. For heterozygous experiments, we used a combination of LP and SB systems. First, a SB plasmid bearing a WT copy of SCN5A was randomly inserted into the genome. A clone of these cells was identified that has an equal level of Nav1.5 in patch clamp experiments to typical LP expression (Figure 2). Next, a second copy of SCN5A bearing WT or variant was incorporated 391 through the LP system. D. Results of flow cytometry after SP and LP integration. Cells express GFP associated with SB integration, and mCherry after LP integration (P5 gate). 

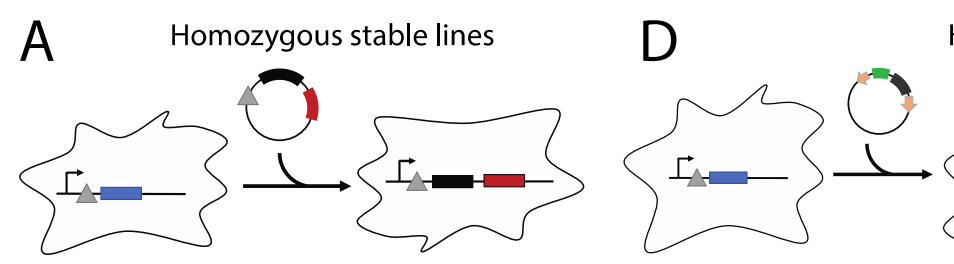

Heterozygous stable lines

B

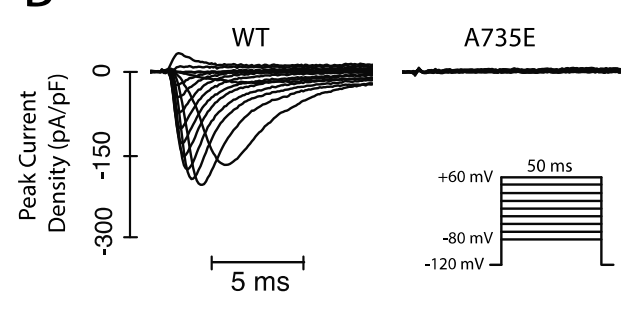

C Homozygous peak current

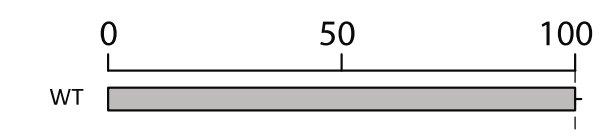

$\mathrm{E}$
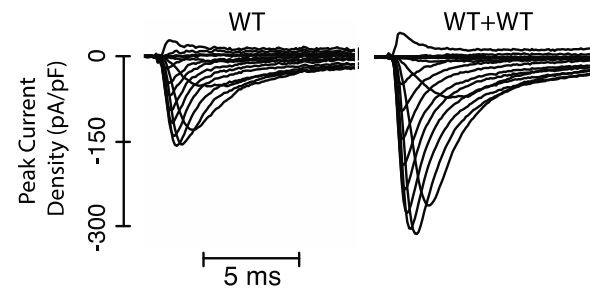

$\mathrm{WT}+\mathrm{A} 735 \mathrm{E}$

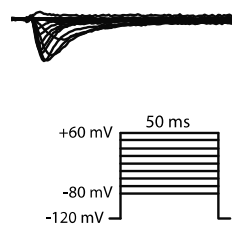

$\mathrm{F}$

Heterozygous peak current density (\% of WT+empty)

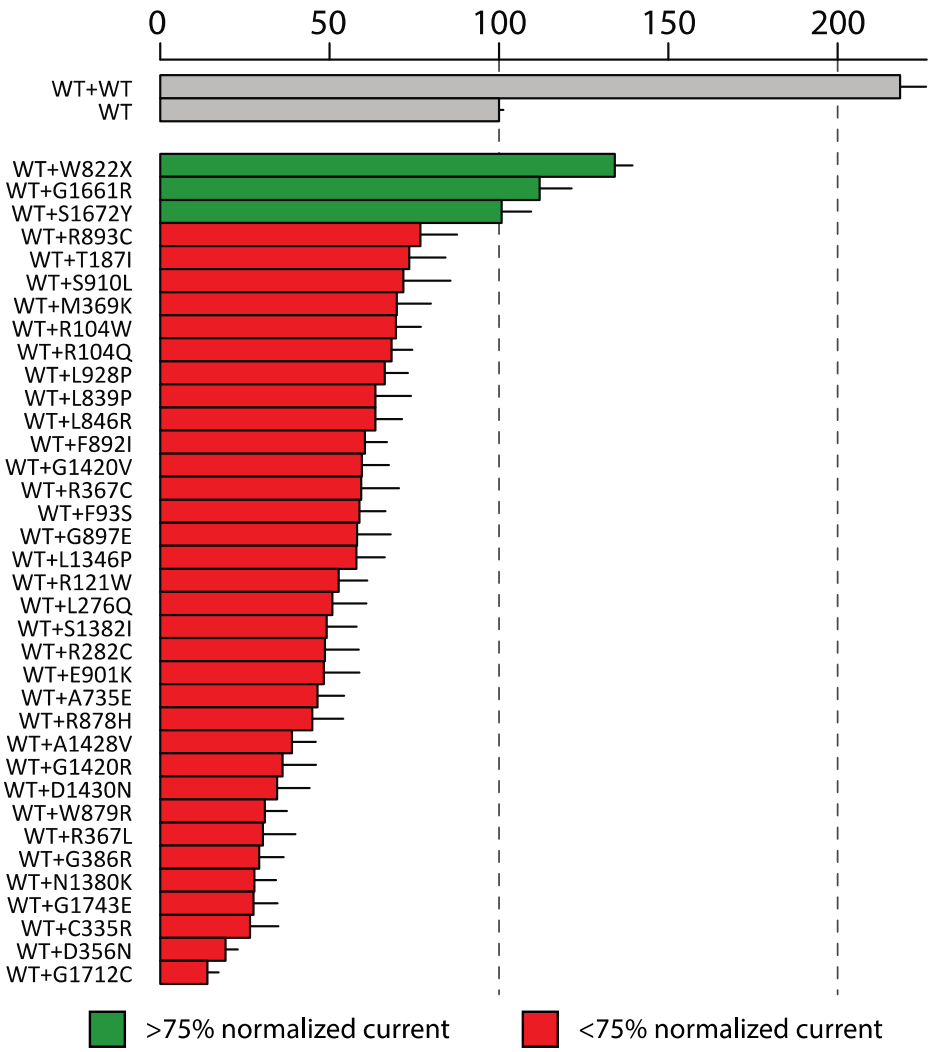

Figure 2. Measurement of loss-of-function homozygous and heterozygous peak current.

A. Introduction of SCN5A variants into LP-SCN5A HEK cells. For full details see Figure 1. 
399 C. Measurement of homozygous peak current density in 35 SCN5A missense variants and one

400 nonsense variant (normalized to WT). Mean \pm standard errors. 11-67 cells were studied per

401 variant.

402 D. Heterozygous LP-SB-SCN5A cell lines. For full details see Figure 1.

403 E. Representative raw peak current densities in a single transfected WT, dually integrated

404 WT+WT, and WT+A735E cell.

405 F. Peak current density measurements for 35 SCN5A missense variants and one nonsense

406 variant in expression with WT SCN5A (normalized to single WT). Mean \pm standard errors. 27-

407164 cells were studied per variant.

408

409

410

411

412

413

414 
A

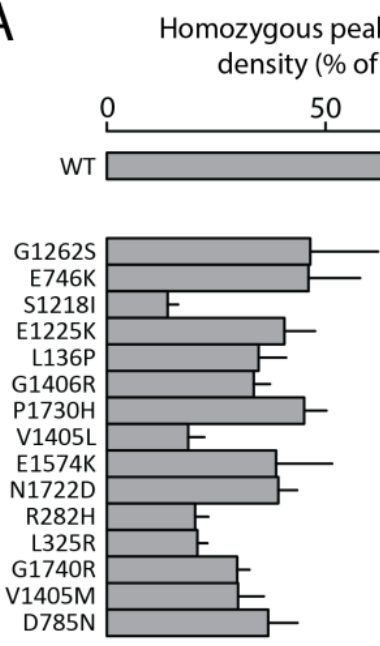

B

Heterozygous peak current

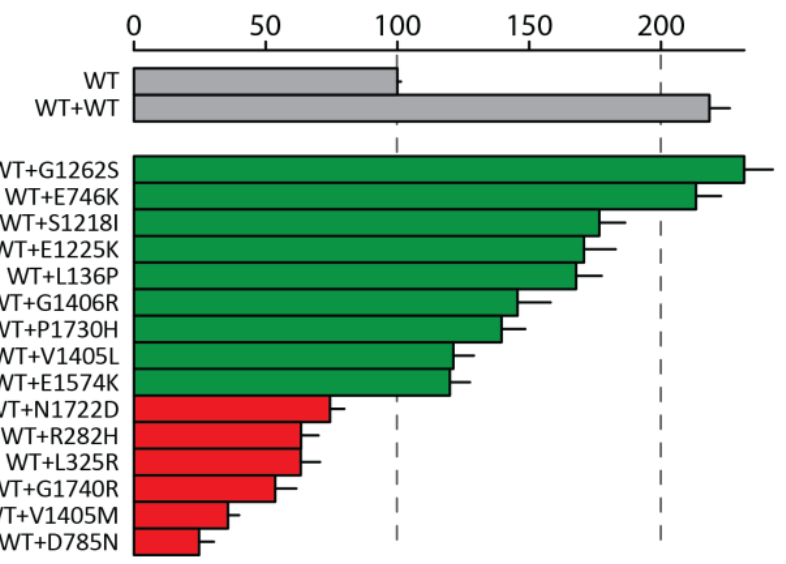

$>75 \%$ normalized current

$<75 \%$ normalized current

Figure 3. Some partial loss-of-function variants have a dominant negative effect.

417 A. Measurement of homozygous peak current density in 15 partial LoF SCN5A variants

418 (normalized to WT). Mean \pm standard errors. 8-36 cells were studied per variant.

419 B. Measurement of heterozygous peak current density in 15 partial LoF SCN5A variants

420 (normalized to WT). Mean \pm standard errors. 27-53 cells were studied per variant. 
A

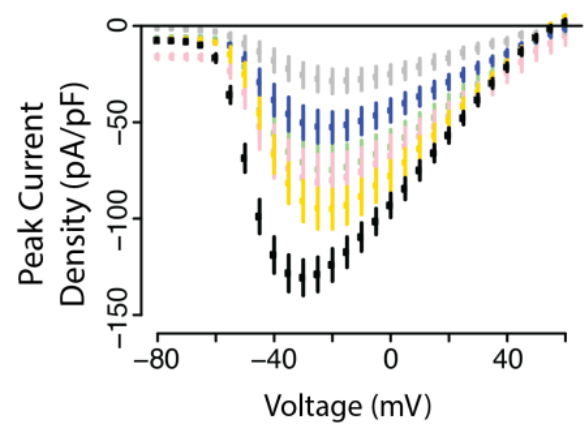

C Voltage Half Activation

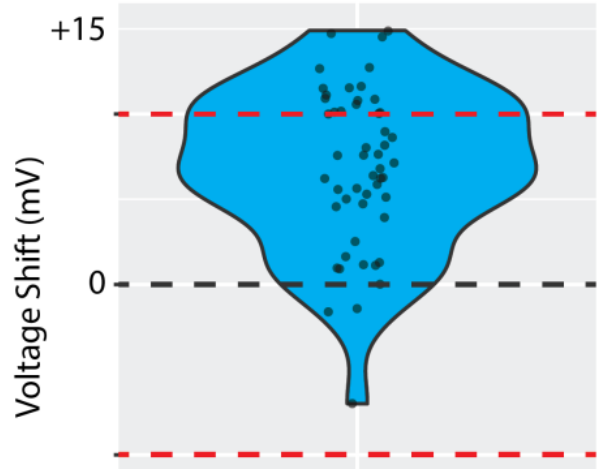

$-15$

Missense Variants

$\mathrm{E}$

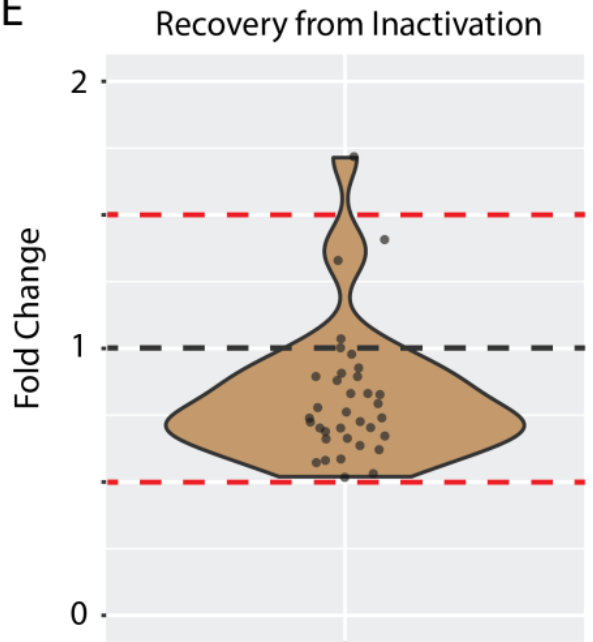

Missense Variants

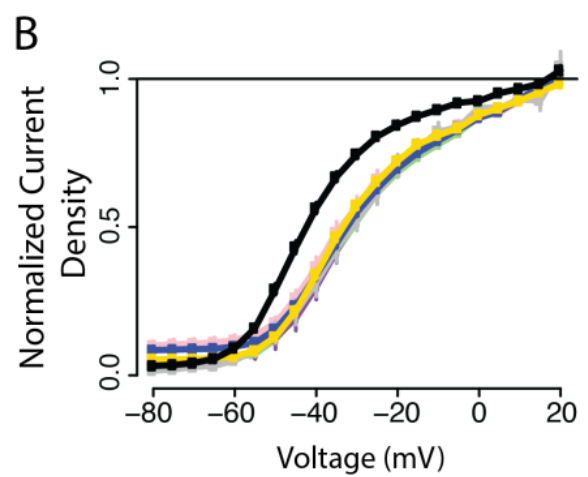

D Voltage Half Inactivation

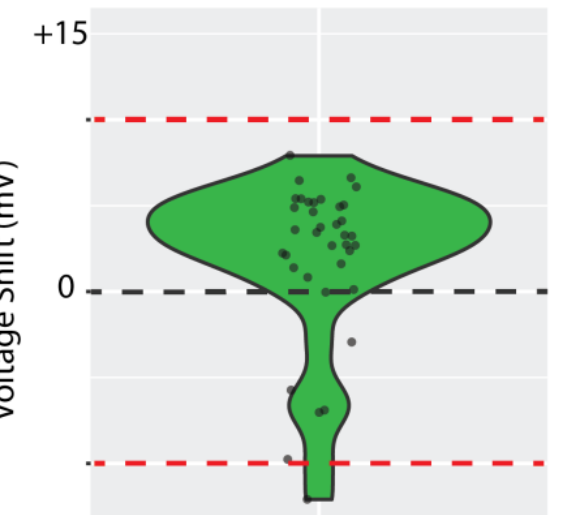

$-15$

Missense Variants

$\mathrm{F}$

Late Current Ratio

$2 \%$.

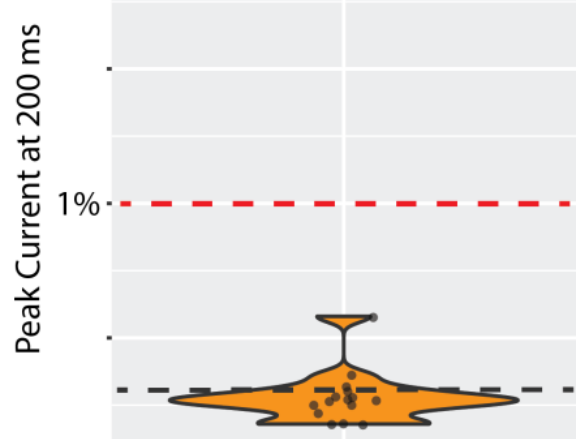

$0 \%$.

Missense Variants 
425 A. Current-voltage plot of WT (black) and 5 missense SCN5A variants with large shifts in

426 voltage of half activation: A735E (light green), R121W (pink), D785N (grey), A1428V (blue),

$427 \quad$ F892l (gold).

428 B. Raw voltage half activation curve for WT and 5 missense SCN5A variants (variants and color

429 same as in A). B-D) WT indicated with black line and abnormal cutoffs indicated with red lines.

430 For B-D only variants with at least 10 qualifying cells meeting quality control criteria were

431 analyzed.

432 C. Voltage of half activation shift of all missense variants compared to WT.

433 D. Voltage of half inactivation shift of all missense variants compared to WT.

434 E. Time of 50\% recovery from inactivation measured in fold change for all missense variants

435 normalized to WT.

436 F. Late current percentage (\% of peak current) measured at $200 \mathrm{~ms}$ for all missense variants

437 compared to WT. 


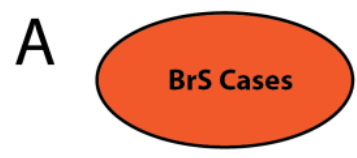

300 Missense Variants 411 BrS Carriers

1483 gnomAD Carriers 11.0 OR

127 Haploins. Variants 153 BrS Carriers 48 gnomAD Carriers 118 OR
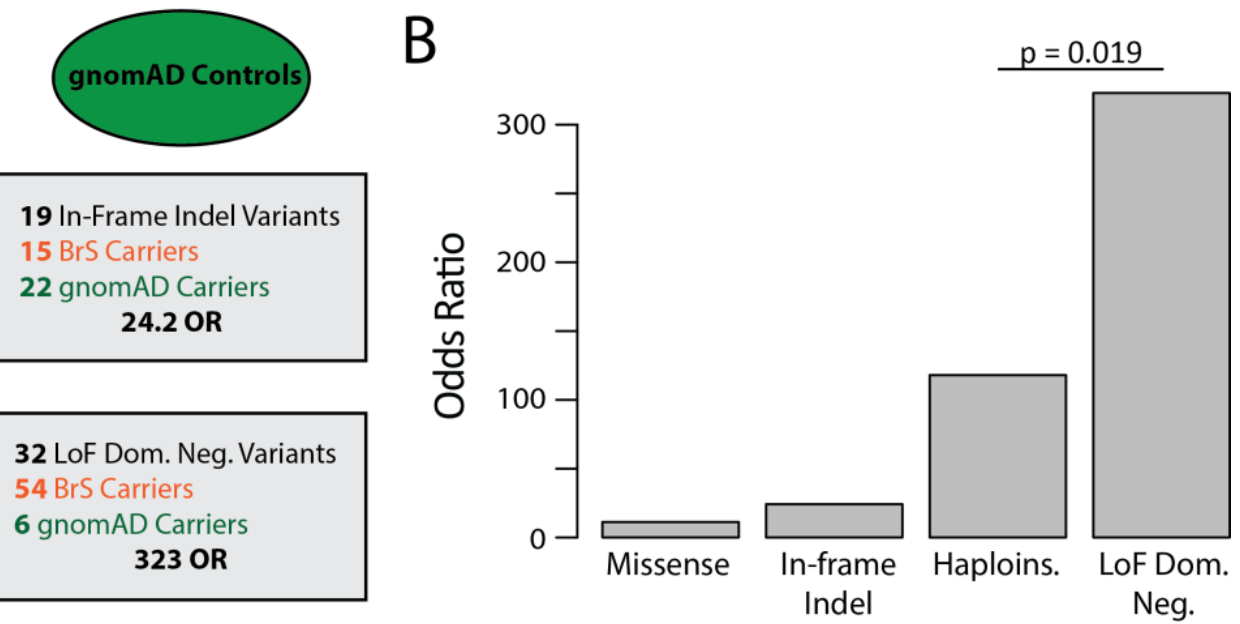

441 Figure 5. Case-Control analysis by variant class

442 A. Case-control breakdown by data source stratified by variant class. BrS cases are shown in

443 red, with putative gnomAD controls shown in green. Haploins. Indicates nonsense, splice, and

444 frameshift variants. Odds ratios are calculated for each variant class.

B. Barplot of BrS odds ratios by variant class. 


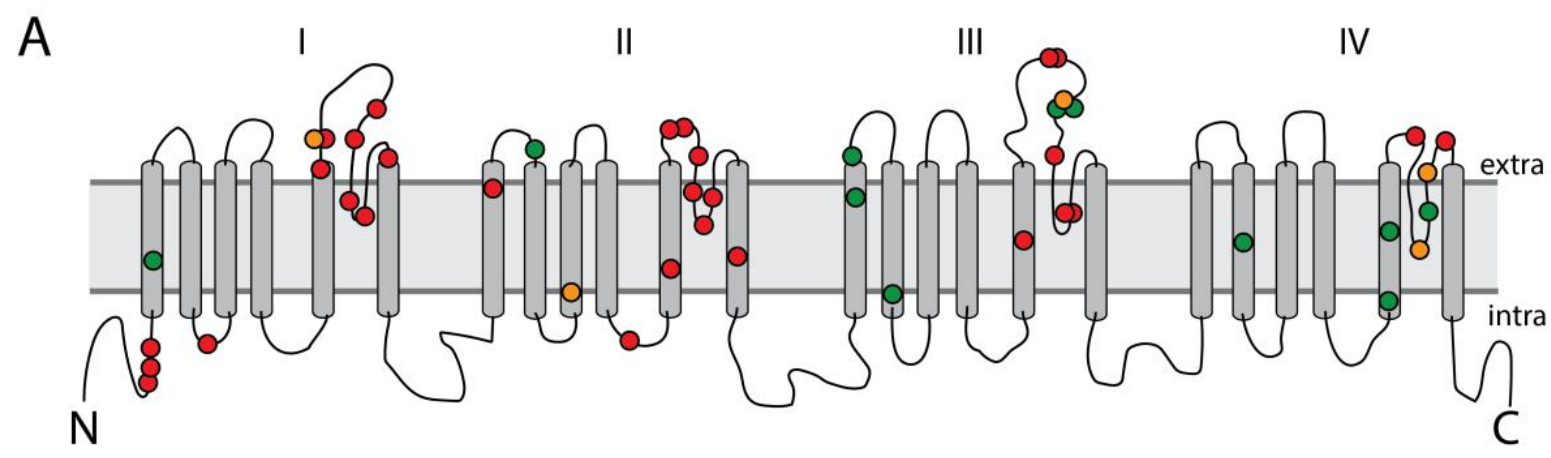

LoF Dominant Negative $\bigcirc$ Partial LoF Dominant Negative

Not Dominant Negative

B

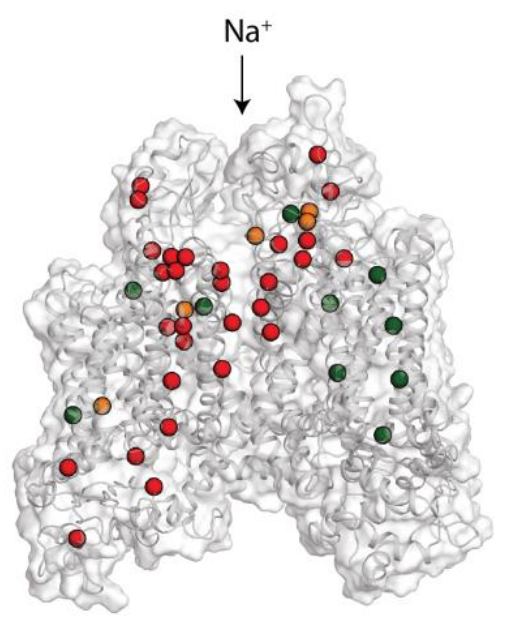

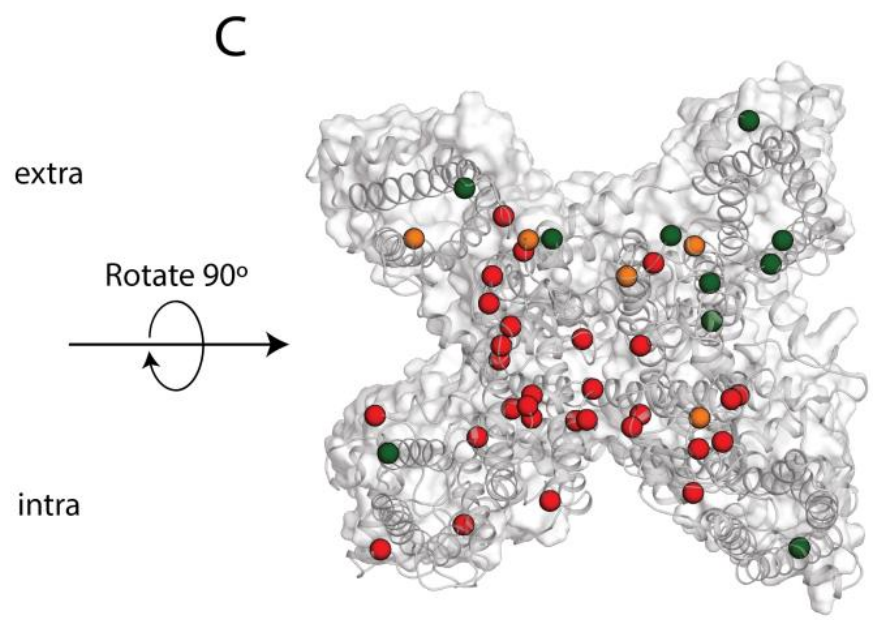

Figure 6. Structural distribution of dominant negative variants.

459 A. Locations of dominant negative variants throughout Nav1.5 in 2D channel rendering. Red

460 indicated LoF dominant negative, orange partial LoF dominant negative, and green non-

461 dominant negative missense variants. Extra: extracellular, intra: intracellular.

462 B. Side view of Nav1.5 protein with overlaid variant distribution.

463 C. Top view of Nav1.5 protein with overlaid variant distribution. 


\section{References}

1. Mizusawa Y, Wilde AA. Brugada syndrome. Circ Arrhythm Electrophysiol. 2012;5(3):60616.

2. Kapplinger JD, Tester DJ, Alders M, Benito B, Berthet M, Brugada J, et al. An international compendium of mutations in the SCN5A-encoded cardiac sodium channel in patients referred for Brugada syndrome genetic testing. Heart Rhythm. 2010;7(1):33-46. 3. Hosseini SM, Kim R, Udupa S, Costain G, Jobling R, Liston E, et al. Reappraisal of Reported Genes for Sudden Arrhythmic Death: Evidence-Based Evaluation of Gene Validity for Brugada Syndrome. Circulation. 2018;138(12):1195-205.

4. Gui J, Wang T, Jones RP, Trump D, Zimmer T, Lei M. Multiple loss-of-function mechanisms contribute to SCN5A-related familial sick sinus syndrome. PLoS One. 2010;5(6):e10985.

5. Bezzina CR, Rook MB, Groenewegen WA, Herfst $L$, van der Wal AC, Lam J, et al. Compound heterozygosity for mutations (W156X and R225W) in SCN5A associated with severe cardiac conduction disturbances and degenerative changes in the conduction system. Circ Res. 2003;92(2):159-68.

6. Kroncke BM, Glazer AM, Smith DK, Blume JD, Roden DM. SCN5A (Na(V)1.5) Variant Functional Perturbation and Clinical Presentation: Variants of a Certain Significance. Circ Genom Precis Med. 2018;11(5):e002095.

7. Pan X, Li Z, Jin X, Zhao Y, Huang G, Huang X, et al. Comparative structural analysis of human $\mathrm{Na}(\mathrm{v}) 1.1$ and $\mathrm{Na}(\mathrm{v}) 1.5$ reveals mutational hotspots for sodium channelopathies. Proc Natl Acad Sci U S A. 2021;118(11).

8. Clatot J, Hoshi M, Wan X, Liu H, Jain A, Shinlapawittayatorn K, et al. Voltage-gated sodium channels assemble and gate as dimers. Nat Commun. 2017;8(1):2077.

9. Clatot J, Zheng Y, Girardeau A, Liu H, Laurita KR, Marionneau C, et al. Mutant voltagegated $\mathrm{Na}(+)$ channels can exert a dominant negative effect through coupled gating. Am J Physiol Heart Circ Physiol. 2018;315(5):H1250-h7.

10. Keller DI, Rougier JS, Kucera JP, Benammar N, Fressart V, Guicheney P, et al. Brugada syndrome and fever: genetic and molecular characterization of patients carrying SCN5A mutations. Cardiovasc Res. 2005;67(3):510-9.

11. Doisne N, Grauso M, Mougenot N, Clergue M, Souil C, Coulombe A, et al. In vivo Dominant-Negative Effect of an SCN5A Brugada Syndrome Variant. Front Physiol. 2021;12:661413.

\section{Walsh R, Lahrouchi N, Tadros R, Kyndt F, Glinge C, Postema PG, et al. Enhancing rare} variant interpretation in inherited arrhythmias through quantitative analysis of consortium disease cohorts and population controls. Genet Med. 2021;23(1):47-58.

13. Karczewski KJ, Francioli LC, Tiao G, Cummings BB, Alföldi J, Wang Q, et al. The mutational constraint spectrum quantified from variation in 141,456 humans. Nature. 2020;581(7809):434-43.

14. Glazer AM, Wada Y, Li B, Muhammad A, Kalash OR, O'Neill MJ, et al. High-Throughput Reclassification of SCN5A Variants. Am J Hum Genet. 2020;107(1):111-23. 
15. Matreyek KA, Stephany JJ, Chiasson MA, Hasle N, Fowler DM. An improved platform for functional assessment of large protein libraries in mammalian cells. Nucleic Acids Res. 2020;48(1):e1.

512 16. Matreyek KA, Stephany JJ, Fowler DM. A platform for functional assessment of large variant libraries in mammalian cells. Nucleic Acids Res. 2017;45(11):e102. 17. Kowarz E, Löscher D, Marschalek R. Optimized Sleeping Beauty transposons rapidly generate stable transgenic cell lines. Biotechnol J. 2015;10(4):647-53. 18. Mátés L, Chuah MK, Belay E, Jerchow B, Manoj N, Acosta-Sanchez A, et al. Molecular evolution of a novel hyperactive Sleeping Beauty transposase enables robust stable gene transfer in vertebrates. Nat Genet. 2009;41(6):753-61. 19. Whiffin N, Minikel E, Walsh R, O'Donnell-Luria AH, Karczewski K, Ing AY, et al. Using high-resolution variant frequencies to empower clinical genome interpretation. Genet Med. 2017;19(10):1151-8.

20. UniProt: the universal protein knowledgebase in 2021. Nucleic Acids Res. 2021;49(D1):D480-d9.

21. Leaver-Fay A, Tyka M, Lewis SM, Lange OF, Thompson J, Jacak R, et al. ROSETTA3: an object-oriented software suite for the simulation and design of macromolecules. Methods Enzymol. 2011;487:545-74.

22. Shen H, Liu D, Wu K, Lei J, Yan N. Structures of human $\mathrm{Na(v)1.7} \mathrm{channel} \mathrm{in} \mathrm{complex} \mathrm{with}$ auxiliary subunits and animal toxins. Science. 2019;363(6433):1303-8. modulation of voltage-gated sodium channels by animal toxins. Science. 2018;362(6412). 24. Li Z, Jin X, Wu T, Huang G, Wu K, Lei J, et al. Structural Basis for Pore Blockade of the Human Cardiac Sodium Channel Na(v) 1.5 by the Antiarrhythmic Drug Quinidine*. Angew Chem Int Ed Engl. 2021;60(20):11474-80.

25. Clatot J, Ziyadeh-Isleem A, Maugenre S, Denjoy I, Liu H, Dilanian G, et al. Dominantnegative effect of SCN5A N-terminal mutations through the interaction of $\mathrm{Na}(\mathrm{v}) 1.5 \alpha$-subunits. Cardiovasc Res. 2012;96(1):53-63.

26. Poelzing S, Forleo C, Samodell M, Dudash L, Sorrentino S, Anaclerio M, et al. SCN5A polymorphism restores trafficking of a Brugada syndrome mutation on a separate gene. Circulation. 2006;114(5):368-76.

27. Baroudi G, Napolitano C, Priori SG, Del Bufalo A, Chahine M. Loss of function associated with novel mutations of the SCN5A gene in patients with Brugada syndrome. Can J Cardiol.

543 28. Veitia RA, Birchler JA. Dominance and gene dosage balance in health and disease: why 544 levels matter! J Pathol. 2010;220(2):174-85.

545 29. Wang Z, Vermij SH, Sottas V, Shestak A, Ross-Kaschitza D, Zaklyazminskaya EV, et al. 546 Calmodulin binds to the N-terminal domain of the cardiac sodium channel $\mathrm{Na}(\mathrm{v}) 1.5$. Channels 547 (Austin). 2020;14(1):268-86.

548 30. Ishikawa T, Kimoto H, Mishima H, Yamagata K, Ogata S, Aizawa Y, et al. Functionally 549 validated SCN5A variants allow interpretation of pathogenicity and prediction of lethal events 550 in Brugada syndrome. Eur Heart J. 2021;42(29):2854-63. 
551 31. Meregalli PG, Tan HL, Probst V, Koopmann TT, Tanck MW, Bhuiyan ZA, et al. Type of 552 SCN5A mutation determines clinical severity and degree of conduction slowing in loss-of-

553 function sodium channelopathies. Heart Rhythm. 2009;6(3):341-8.

554 32. Obergrussberger A, Friis S, Brüggemann A, Fertig N. Automated patch clamp in drug 555 discovery: major breakthroughs and innovation in the last decade. Expert Opin Drug Discov. 556 2021;16(1):1-5.

557 33. Vanoye CG, Desai RR, Fabre KL, Gallagher SL, Potet F, DeKeyser JM, et al. High-

558 Throughput Functional Evaluation of KCNQ1 Decrypts Variants of Unknown Significance. Circ 559 Genom Precis Med. 2018;11(11):e002345.

560 34. Glazer AM, Davogustto G, Shaffer CM, Vanoye CG, Desai RR, Farber-Eger EH, et al. 561 Arrhythmia variant associations and reclassifications in the eMERGE-III sequencing study. 562 medRxiv. 2021:2021.03.30.21254549.

563 35. Ng CA, Perry MD, Liang W, Smith NJ, Foo B, Shrier A, et al. High-throughput phenotyping 564 of heteromeric human ether-à-go-go-related gene potassium channel variants can discriminate 565 pathogenic from rare benign variants. Heart Rhythm. 2020;17(3):492-500.

566 36. Ng C-A, Ullah R, Farr J, Hill AP, Kozek KA, Vanags LR, et al. A Massively Parallel Trafficking 567 Assay Accurately Predicts Loss of Channel Function in <em $>\mathrm{KCNH} 2</$ em $>$ Variants. bioRxiv.

568 2021:2021.07.10.451881.

569 37. Xu Z, Fulop Z, Wu G, Pone EJ, Zhang J, Mai T, et al. 14-3-3 adaptor proteins recruit AID to 5705 '-AGCT-3'-rich switch regions for class switch recombination. Nat Struct Mol Biol.

$5712010 ; 17(9): 1124-35$.

572 38. Zheng Y, Wan X, Yang D, Ramirez-Navarro A, Liu H, Fu JD, et al. A Heart Failure-

573 Associated SCN5A Splice Variant Leads to a Reduction in Sodium Current Through Coupled-

574 Gating With the Wild-Type Channel. Front Physiol. 2021;12:661429.

575 39. Nguyen K, Wang Y, England WE, Chaput JC, Spitale RC. Allele-Specific RNA Knockdown

576 with a Biologically Stable and Catalytically Efficient XNAzyme. J Am Chem Soc.

577 2021;143(12):4519-23.

578 40. Dotzler SM, Kim CSJ, Gendron WAC, Zhou W, Ye D, Bos JM, et al. Suppression-

579 Replacement KCNQ1 Gene Therapy for Type 1 Long QT Syndrome. Circulation. 2021.

580 41. Bezzina CR, Barc J, Mizusawa Y, Remme CA, Gourraud JB, Simonet F, et al. Common

581 variants at SCN5A-SCN10A and HEY2 are associated with Brugada syndrome, a rare disease with 582 high risk of sudden cardiac death. Nat Genet. 2013;45(9):1044-9. 
Table S1. Variant currents and case-control counts.

587

\begin{tabular}{|c|c|c|c|c|c|c|c|c|c|}
\hline Variant & $\begin{array}{c}\text { Homozygous } \\
\text { Peak } \\
\text { Current } \\
\text { Density } \\
\end{array}$ & S.E. & Cells & $\begin{array}{c}\text { Heterozygous } \\
\text { Peak Current } \\
\text { Density }\end{array}$ & S.E. & Cells & $\begin{array}{c}\text { gnomAD } \\
\text { Count }\end{array}$ & $\begin{array}{c}\text { gnomAD } \\
\text { MAF }\end{array}$ & $\begin{array}{l}\text { Walsh } \\
\text { Count }\end{array}$ \\
\hline WT & 100 & 1.3 & 2196 & 100 & 1.3 & 2196 & - & - & - \\
\hline $\mathrm{WT}+\mathrm{WT}$ & - & - & - & 218.4 & 7.7 & 199 & - & - & - \\
\hline G1262S & 46.5 & 15.5 & 10 & 231.6 & 10.8 & 47 & 8 & 2.83E-05 & 3 \\
\hline E746K & 46.1 & 11.8 & 9 & 213.3 & 9.5 & 45 & 6 & $2.14 \mathrm{E}-05$ & 5 \\
\hline S1218I & 13.9 & 2.4 & 19 & 176.6 & 9.8 & 47 & 1 & $4.02 \mathrm{E}-06$ & 0 \\
\hline E1225K & 40.6 & 7 & 19 & 170.8 & 12 & 43 & 1 & $4.01 \mathrm{E}-06$ & 5 \\
\hline L136P & 34.7 & 6.3 & 16 & 167.8 & 9.7 & 41 & 0 & 0 & 2 \\
\hline G1406R & 33.6 & 3.7 & 18 & 145.6 & 12.5 & 35 & 0 & 0 & 3 \\
\hline $\mathrm{P} 1730 \mathrm{H}$ & 45.1 & 5.1 & 31 & 139.5 & 9 & 47 & 0 & 0 & 0 \\
\hline W822X & 4.7 & 0.9 & 16 & 134.2 & 5.2 & 164 & 0 & 0 & 0 \\
\hline V1405L & 18.6 & 3.7 & 15 & 121.2 & 7.8 & 53 & 0 & 0 & 4 \\
\hline E1574K & 38.7 & 12.8 & 8 & 119.9 & 7.6 & 46 & 0 & 0 & 3 \\
\hline G1661R & 5.6 & 1.5 & 19 & 112 & 9.4 & 44 & 0 & 0 & 3 \\
\hline S1672Y & 1 & 0.6 & 18 & 100.8 & 8.7 & 47 & 0 & 0 & 1 \\
\hline R893C & 8.2 & 0.9 & 48 & 76.8 & 10.8 & 52 & 3 & 1.06E-05 & 2 \\
\hline N1722D & 39.2 & 4.3 & 26 & 74.4 & 5.4 & 43 & 0 & 0 & 1 \\
\hline T187I & 0.2 & 0.1 & 42 & 73.5 & 10.7 & 39 & 0 & 0 & 1 \\
\hline S910L & 1.2 & 0.2 & 19 & 71.8 & 13.9 & 35 & 1 & 3.99E-06 & 3 \\
\hline M369K & 3.7 & 0.9 & 22 & 69.8 & 10.1 & 51 & 0 & 0 & 2 \\
\hline R104W & 0.5 & 0.2 & 24 & 69.6 & 7.3 & 43 & 1 & $4.01 \mathrm{E}-06$ & 3 \\
\hline R104Q & 0.4 & 0.2 & 22 & 68.3 & 6.1 & 34 & 0 & 0 & 3 \\
\hline L928P & 1.4 & 0.9 & 27 & 66.3 & 6.8 & 47 & 0 & 0 & 1 \\
\hline L839P & 3.1 & 2.2 & 20 & 63.5 & 10.5 & 53 & 0 & 0 & 1 \\
\hline L846R & 0.3 & 0.2 & 43 & 63.5 & 7.9 & 35 & 0 & 0 & 0 \\
\hline $\mathrm{R} 282 \mathrm{H}$ & 20.2 & 3 & 16 & 63.4 & 6.6 & 44 & 4 & $1.60 \mathrm{E}-05$ & 8 \\
\hline L325R & 20.7 & 2.3 & 36 & 63.3 & 7.3 & 49 & 0 & 0 & 0 \\
\hline F892I & 0.9 & 0.7 & 23 & 60.4 & 6.5 & 51 & 0 & 0 & 1 \\
\hline G1420V & 0 & 0 & 11 & 59.5 & 8 & 52 & 0 & 0 & 1 \\
\hline R367C & 0.6 & 0.3 & 25 & 59.3 & 11.2 & 54 & 3 & 1.07E-05 & 3 \\
\hline F93S & 0.2 & 0.2 & 15 & 58.8 & 7.7 & 53 & 0 & 0 & 1 \\
\hline G897E & 0.8 & 0.3 & 16 & 58.1 & 9.9 & 38 & 0 & 0 & 0 \\
\hline L1346P & 2.1 & 0.9 & 15 & 57.9 & 8.4 & 53 & 0 & 0 & 1 \\
\hline G1740R & 29.8 & 2.8 & 20 & 53.6 & 8 & 27 & 0 & 0 & 1 \\
\hline R121W & 0.7 & 0.3 & 40 & 52.7 & 8.4 & 36 & 0 & 0 & 3 \\
\hline L276Q & 1.1 & 0.8 & 14 & 50.8 & 10.1 & 53 & 0 & 0 & 2 \\
\hline S1382I & 4.5 & 1 & 29 & 49.1 & 8.9 & 47 & 0 & 0 & 1 \\
\hline $\mathrm{R} 282 \mathrm{C}$ & 1.4 & 0.3 & 67 & 48.6 & 10 & 55 & 0 & 0 & 2 \\
\hline E901K & 3.3 & 0.6 & 16 & 48.3 & 10.5 & 46 & 0 & 0 & 6 \\
\hline A735E & 1.3 & 0.9 & 12 & 46.4 & 7.9 & 39 & 0 & 0 & 0 \\
\hline R878H & 0.2 & 0.1 & 38 & 44.9 & 9.1 & 39 & 0 & 0 & 3 \\
\hline A1428V & 0.3 & 0.3 & 24 & 38.9 & 7 & 53 & 0 & 0 & 1 \\
\hline G1420R & 2.5 & 1.2 & 16 & 36.1 & 9.9 & 50 & 0 & 0 & 2 \\
\hline V1405M & 30 & 5.9 & 14 & 35.7 & 4.2 & 38 & 0 & 0 & 5 \\
\hline D1430N & 0.4 & 0.1 & 57 & 34.5 & 9.6 & 28 & 0 & 0 & 0 \\
\hline W879R & 0 & 0 & 43 & 30.9 & 6.5 & 46 & 0 & 0 & 0 \\
\hline R367L & 0 & 0 & 39 & 30.3 & 9.6 & 46 & 0 & 0 & 1 \\
\hline G386R & 1.5 & 0.9 & 11 & 29.2 & 7.2 & 52 & 0 & 0 & 0 \\
\hline N1380K & 0.1 & 0.1 & 25 & 27.8 & 6.4 & 42 & 0 & 0 & 1 \\
\hline G1743E & 1 & 0.4 & 11 & 27.5 & 7.1 & 37 & 0 & 0 & 5 \\
\hline C335R & 0 & 0 & 24 & 26.5 & 8.4 & 27 & 0 & 0 & 1 \\
\hline D785N & 36.9 & 6.7 & 27 & 24.7 & 5.6 & 33 & 0 & 0 & 0 \\
\hline D356N & 1.4 & 0.3 & 16 & 19.3 & 3.6 & 45 & 1 & $4.02 \mathrm{E}-06$ & 5 \\
\hline G1712C & 8.3 & 2.4 & 17 & 13.9 & 3.3 & 38 & 0 & 0 & 0 \\
\hline
\end{tabular}


Table S2 - Primers used in this Study.

591

\begin{tabular}{|c|c|c|}
\hline Variant & Name & Sequence \\
\hline F93S & ag738 & CTATAGCACCCAAAAGACTTCCATCGTACTGAATAAAGGCA \\
\hline $\mathrm{R} 104 \mathrm{Q}$ & $\operatorname{ag} 1122$ & GGCAAGACCATCTTCCAGTTCAGTGCCACCAAC \\
\hline R104W & ag885 & GGCAAGACCATCTTCTGGTTCAGTGCCACCA \\
\hline $\mathrm{R} 121 \mathrm{~W}$ & ag655 & CTTCCACCCCATCTGGAGAGCGGCTGT \\
\hline L136P & $\operatorname{ag} 740$ & CTCGCTCTTCAACATGCCCATCATGTGCACCATCC \\
\hline T187I & $\operatorname{ag} 1123$ & CCTGCACGCATTCATTTTCCTTCGGGACC \\
\hline L276Q & $\operatorname{ag} 742$ & СTCTTCATGGGCAACCAAAGGCACAAGTGCGTG \\
\hline $\mathrm{R} 282 \mathrm{C}$ & ag729 & GGCACAAGTGCGTGTGCAACTTCACAGCG \\
\hline $\mathrm{R} 282 \mathrm{H}$ & $\operatorname{ag} 1124$ & GCACAAGTGCGTGCACAACTTCACAGCGC \\
\hline C335R & ag785 & GACGCTGGGACACGTCCGGAGGGCT \\
\hline D356N & ag1125 & GGCTACACCAGCTTCAATTCCTTTGCCTGGG \\
\hline R367C & $\operatorname{ag} 778$ & TTTCTTGCACTCTTCCTCCTGATGACGCAGGAC \\
\hline R367L & ag665 & СTTTCTTGCACTCTTCTGCCTGATGACGCAGGA \\
\hline M369K & $\operatorname{ag} 743$ & CTCTTCCGCCTGAAGACGCAGGACTGC \\
\hline G386R & ag745 & AGACCCTCAGGTCCGCAAGGAAGATCTACATG \\
\hline A735E & ag746 & CAACACACTCTTCATGGAGCTGGAGCACTACAACA \\
\hline E746K & ag669 & GCGGCCGCGAATTCAAGGAGATGCTGCA \\
\hline $\mathrm{D} 785 \mathrm{~N}$ & $\operatorname{ag} 748$ & AGGGCTGGAACATCTTCAACAGCATCATCGTCATC \\
\hline W822X & ag68 & GCTGGCCAAATCATGACCCACCCTGAACACA \\
\hline L893P & $\operatorname{ag} 749$ & CAGTGGGGGCACCGGGGAACCTGAC \\
\hline L846R & ag1126 & AACCTGACACTGGTGCGTGCCATCATCGTGTTC \\
\hline $\mathrm{R} 878 \mathrm{H}$ & ag1127 & GGCCTGCTGCCTCACTGGCACATGATG \\
\hline W879R & ag798 & CCTGCTGCCTCGCAGGCACATGATGGA \\
\hline F8921 & $\operatorname{ag} 750$ & GCCTTCCTCATCATCATCCGCATCCTCTGTG \\
\hline $\mathrm{R} 893 \mathrm{H}$ & ag1128 & СТTССТСАТСАТСTTCСАСАTССTСTGTGGAGAGT \\
\hline G897E & ag1129 & TCCGCATCCTCTGTGAAGAGTGGATCGAGAC \\
\hline E901K & ag678 & CTGTGGAGAGTGGATCAAGACCATGTGGGACTG \\
\hline S910L & $\operatorname{ag} 1130$ & GGACTGCATGGAGGTGTTGGGGCAGTC \\
\hline L928P & ag782 & TATGGTCATTGGCAACCCTGTGGTCCTGAATCTCT \\
\hline S1218I & $\operatorname{ag} 1131$ & TCATGATCCTACTCATCAGTGGAGCGCTGGC \\
\hline $\mathrm{E} 1225 \mathrm{~K}$ & ag687 & GGAGCGCTGGCCTTCAAGGACATCTACCTAG \\
\hline G1262S & ag690 & TCAAGTGGGTGGCCTACAGCTTCAAGAAGTACTTC \\
\hline L1346P & ag754 & CTGCCTCATCTTCTGGCCCATCTTCAGCATCATGG \\
\hline N1380K & ag755 & TTTGAACTACACCATCGTGAACAAAAAGAGCCAGTGTG \\
\hline S1382l & ag795 & CTACACCATCGTGAACAACAAGATCCAGTGTGAGTC \\
\hline V1405M & ag757 & AAAGTCAACTTTGACAACATGGGGGCCGGGTAC \\
\hline G1406R & $\operatorname{ag} 1132$ & CTTTGACAACGTGCGGGCCGGGTACCT \\
\hline G1408R & ag1133 & ACGTGGGGGCCAGGTACCTGGCC \\
\hline
\end{tabular}


bioRxiv preprint doi: https://doi.org/10.1101/2021.09.22.461398; this version posted September 24, 2021. The copyright holder for this preprint (which was not certified by peer review) is the author/funder, who has granted bioRxiv a license to display the preprint in perpetuity. It is made available under aCC-BY-ND 4.0 International license.

593 Table S3. Case-control analysis.

594

\begin{tabular}{ccccccc}
\hline Class & $\begin{array}{c}\text { \# of } \\
\text { variants }\end{array}$ & $\begin{array}{c}\text { BrS } \\
\text { cohort } \\
\text { count }\end{array}$ & $\begin{array}{c}\text { gnomAD } \\
\text { count }\end{array}$ & $\begin{array}{c}\text { gnomAD } \\
\text { AF }\end{array}$ & $\begin{array}{c}\text { BrS : } \\
\text { gnomAD } \\
\text { ratio }\end{array}$ & $\begin{array}{c}\text { Odds } \\
\text { Ratio }\end{array}$ \\
\hline All missense & 300 & 411 & 1483 & $5.9 \mathrm{e}-3$ & 0.28 & 11.0 \\
In-frame indel & 19 & 15 & 22 & $8.7 \mathrm{e}-5$ & 0.68 & 24.2 \\
Frameshift+splice & 127 & 153 & 48 & $4.2 \mathrm{e}-4$ & 3.19 & 118 \\
Missense LoF + & 32 & 54 & 6 & $2.3 \mathrm{e}-5$ & 9.0 & 323 \\
Dom. Neg. & 32 & & &
\end{tabular}

595

596 


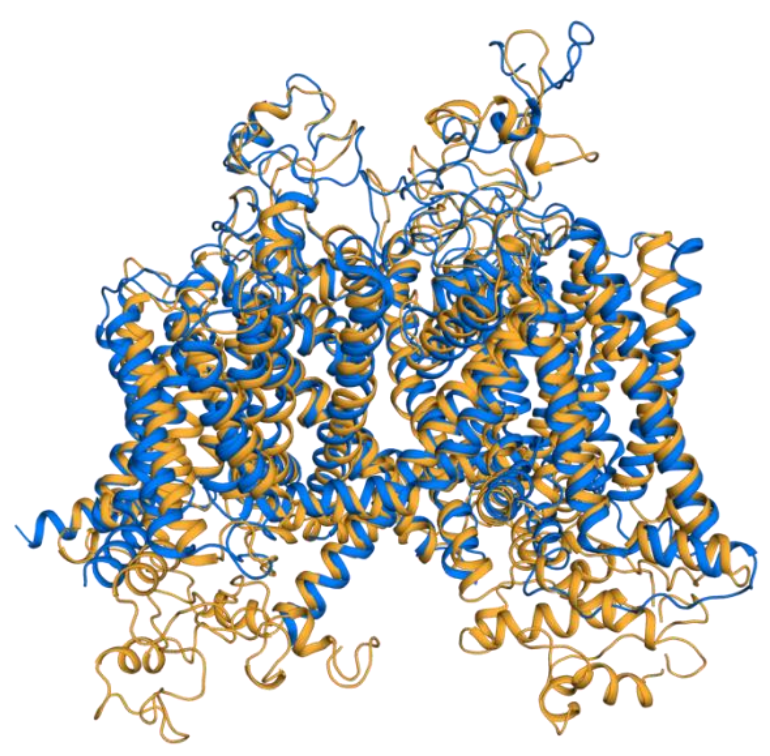

598 Figure S1. Overlay of our Nav1.5 structural model (light orange) with a recently determined

599 cryo-EM structure of Nav1.5 (marine blue), demonstrating that our model is accurate while

600 covering more intracellular residues than the experimental structure (24). 